\title{
Relationships between online health information seeking and psychopathology
}

David Berle ${ }^{1,2 *}$, Vladan Starcevic ${ }^{3}$, Yasser Khazaal ${ }^{4,5}$, Kirupamani Viswasam 6 , Vincent Hede $^{7}$, \& Ryan D. McMullan ${ }^{8}$

1. Discipline of Clinical Psychology, University of Technology Sydney, Sydney, Australia.

2. School of Psychiatry, University of New South Wales, Sydney, Australia.

3. Discipline of Psychiatry, Faculty of Medicine and Health, Sydney Medical School, Nepean Clinical School, University of Sydney, Sydney, Australia.

4. Addiction medicine. Department of Psychiatry. Lausanne University Hospitals, lausanne Switzerland

5. Research Centre, University Institute of Mental Health at Montreal, Quebec, Canada.

6. Department of Psychiatry, Nepean Hospital, Penrith, Australia.

7. Department of Mental Health and Psychiatry, Geneva University Hospitals, Geneva, Switzerland.

8. Australian Institute of Health Innovation, Macquarie University, Sydney, Australia.

Address for correspondence:

David Berle

Discipline of Clinical Psychology

Graduate School of Health

Vicki Sara Building

University of Technology Sydney

67 Thomas Street

Ultimo NSW 2007

Australia

Email: david.berle@uts.edu.au

Ph: +61295144278 


\section{Introduction}

Individuals with higher levels of health anxiety tend to search for health information online more often and for greater amounts of time than others [1]. It is unclear to what extent people with elevated levels of general anxiety, depressive or obsessive-compulsive symptoms engage in online health information seeking (OHIS). Given that health anxiety frequently occurs alongside elevated levels of anxiety, depressive and obsessive-compulsive symptoms, the latter can also be expected to be associated with OHIS. Oh and Song [2] found depressive symptoms to predict OHIS, but did not assess anxiety symptoms. To the best of our knowledge, these relationships have not otherwise been investigated.

High levels of intolerance of uncertainty (IU) have been reported to be related to excessive OHIS [3]. This is understandable, given that individuals with prominent IU may attempt to reduce uncertainty and doubts about their health and symptoms by searching for health information online.

We aimed to determine the proportion of people who have engaged in recent OHIS; ascertain the relationships between OHIS on one hand and IU, health anxiety, anxiety, obsessive-compulsive and depressive symptoms, on the other; and determine whether OHIS is predicted by levels of health anxiety and/or IU, once other conceptually related variables are controlled for.

\section{Methods}

Adults $(N=992)$ were recruited through an online research participation site. The study was approved by the [blinded-for-review] Human Research Ethics Committee and the [blinded-for-review]. The sample was restricted to participants from Australia, Canada, Ireland, New Zealand, the United Kingdom, and the United States of America to reduce linguistic, sociocultural and economic heterogeneity. 
Participants were asked whether or not they had engaged in OHIS in the preceding three months and administered the following questionnaires: the Intolerance of Uncertainty Scale Short Form (IUS-12 [4]); the Obsessive Compulsive Inventory Revised (OCI-R [5]); the Patient Health Questionnaire-9 (PHQ-9, a measure of depressive symptoms [6]); the Patient Health Questionnaire-15 Somatic Symptom Severity Scale (PHQ-15 [7]); the seven anxiety items of the Patient-Reported Outcomes Measurement Information System Emotional Distress-Short Form questionnaire (PROMIS [8]); and the Short Health Anxiety Inventory (SHAI [9]).

\section{Results}

Of the 992 participants in our study $(67.4 \%$ female; $61.5 \%$ tertiary educated; $61.0 \%$ currently living with partner), 751 (75.7\%) reported engaging in OHIS in the preceding 3 months. Demographic characteristics are summarised in Table 1, Supplementary data. 1. People who had engaged in OHIS had significantly higher levels of IU and all the symptoms assessed in the study (Table 1, Supplementary data).

Logistic regression analyses were conducted to identify independent predictors of OHIS. First, PHQ-15 and SHAI scores were entered because searching online is likely to be related to the presence of somatic symptoms (PHQ-15) and health anxiety (SHAI). Next, gender was included and at the final step, the PROMIS, PHQ-9 and OCI-R were entered. Only somatic symptoms showed an independent association with OHIS, such that higher levels of reported somatic symptoms were associated with a greater odds of OHIS $(\mathrm{ExpB}=1.12, p<0.05 ;$ Table1, upper section).

We repeated the analysis with IUS-12 scores entered at the first step instead of SHAI (Table 1, lower section). Only somatic symptoms showed an independent association with 
OHIS, such that higher levels of reported somatic symptoms were associated with a greater odds of OHIS $(\operatorname{ExpB}=1.13, p<0.05)$.

\section{Discussion}

Our results suggest that OHIS is commonplace (76\%), consistent with estimates from previous large samples (72\%; [10]). Participants with a university or higher education were more likely to have engaged in recent OHIS. In line with previous studies [e.g., 1], we found that participants with recent OHIS had higher levels of health anxiety than others. OHIS was also associated with higher levels of all assessed psychopathological variables.

However, only somatic symptoms independently predicted OHIS when each of these other symptom domains were controlled for. Thus, somatic symptoms may (partially) explain the relationship between psychopathology and OHIS. This is broadly consistent with the finding that individuals with chronic medical conditions who are likely to have more severe somatic symptoms, are also more likely to engage in OHIS.

A limitation of our study was that our sample was not necessarily representative. However, our findings appear broadly consistent with the only representative survey we are aware of [10].

In conclusion, our study confirms that OHIS is common. The severity of somatic symptoms was found to be a unique predictor of this behaviour. Medical practitioners can thus expect that patients reporting prominent somatic symptoms are particularly likely to engage in OHIS, regardless of the presence of psychopathology. 


\section{References}

1. Baumgartner, S. E. \& Hartmann, T. The Role of Health Anxiety in Online Health Information Search. Cyberpsych Beh Soc N, 14 (2011) 613-618. doi: 10.1089/cyber.2010.0425

2. Oh, Y. S., \& Song, N. K. Investigating Relationships Between Health-Related Problems and Online Health Information Seeking. CIN: Comput Informatics Nurs 35 (2017) 29-35. doi: 10.1097/CIN.0000000000000234

3. Fergus, T. A. Cyberchondria and intolerance of uncertainty: Examining when individuals experience health anxiety in response to Internet searches for medical information. Cyberpsychol Behav Soc Network, 16 (2013) 735-739. doi: 10.1089/cyber.2012.0671

4. Carleton, R. N., Norton, M. A. P. J., \& Asmundson, G. J. G. Fearing the unknown: A short version of the Intolerance of Uncertainty Scale. J Anxiety Disord, 21 (2007) 105-117. doi: 10.1016/j.janxdis.2006.03.014

5. Foa, E. B., Huppert, J. D., Leiberg, S., Langner, R., Kichic, R., Hajcak, G., \& Salkovskis, P. M. The Obsessive-Compulsive Inventory: development and validation of a short version. Psychol Assess, 14 (2002) 485-496. doi:10.1037/10403590.14.4.485

6. Kroenke, K., Spitzer, R. L., \& Williams, J. B. W. The PHQ-9. J Gen Int Med, 16 (2001) 606-613. doi: 10.1046/j.1525-1497.2001.016009606.x

7. Kroenke, K., Spitzer, R. L., \& Williams, J. B. W. The PHQ-15 : Validity of a New Measure for Evaluating the Severity of Somatic Symptoms. Psychosom Med, 266 (2002) 258-266.

8. Pilkonis, P. A., Choi, S. W., Reise, S. P., Stover, A. M., Riley, W. T., \& Cella, D. 
Item Banks for Measuring Emotional Distress From the Patient-Reported Outcomes Measurement Information System (PROMIS $\left.{ }^{\circledR}\right)$ : Depression, Anxiety, and Anger. Assess, 18 (2011) 263-283. doi:10.1177/1073191111411667

9. Abramowitz, J. S., Deacon, B. J., \& Valentiner, D. P. The Short Health Anxiety Inventory: Psychometric Properties and Construct Validity in a Non-clinical Sample. Cognitive Ther Res, 31 (2007) 871-883. doi: 10.1007/s10608-006-9058-1

10. Fox, S., \& Duggan, M. Health Online 2013. Retrieved March 8, 2018, from http://pewinternet.org/Reports/2013/Health-online.aspx 
Table 1. Logistic regression analysis predicting engagement in online health information seeking $(N=992)$.

\begin{tabular}{|c|c|c|c|c|c|}
\hline \multirow[b]{2}{*}{ Predictor variables } & \multirow{2}{*}{$\begin{array}{c}\text { Unstandardised } \\
\text { B (SE) }\end{array}$} & \multirow{2}{*}{$\begin{array}{c}\text { Wald test (z- } \\
\text { ratio) }\end{array}$} & \multirow[t]{2}{*}{$\operatorname{Exp}(B)$} & \multicolumn{2}{|c|}{$95 \% \mathrm{CI}$} \\
\hline & & & & Lower & Upper \\
\hline \multicolumn{6}{|l|}{ Step 1} \\
\hline PHQ-15 & $0.12(0.02)$ & 33.72 & 1.13 & 1.08 & $1.18^{*}$ \\
\hline SHAI total & $0.01(0.01)$ & 0.90 & 1.01 & 0.99 & 1.04 \\
\hline \multicolumn{6}{|l|}{ Step 2} \\
\hline Gender & $0.27(0.16)$ & 2.76 & 1.31 & 0.95 & 1.79 \\
\hline \multicolumn{6}{|l|}{ Step 3} \\
\hline PHQ-9 & $-0.36(0.21)$ & 2.85 & 0.97 & 0.93 & 1.01 \\
\hline PROMIS total & $0.01(0.02)$ & 0.47 & 1.01 & 0.98 & 1.05 \\
\hline OCI-R & $0.0002(0.008)$ & 0.0005 & 1.00 & 0.98 & 1.02 \\
\hline \multicolumn{6}{|l|}{ Regression 2: } \\
\hline & Unstandardised & Wald test (z- & $\operatorname{Exp}(B)$ & \multicolumn{2}{|c|}{$95 \% \mathrm{CI}$} \\
\hline Predictor variables & $\mathrm{B}(\mathrm{SE})$ & ratio) & & Lower & Upper \\
\hline \multicolumn{6}{|l|}{ Step 1} \\
\hline PHQ-15 & $0.13(0.02)$ & 41.78 & 1.14 & 1.09 & $1.18^{*}$ \\
\hline IUS-12 & $0.005(0.008)$ & 0.38 & 1.01 & 0.99 & 1.02 \\
\hline \multicolumn{6}{|l|}{ Step 2} \\
\hline Gender & $0.27(0.16)$ & 2.83 & 1.31 & 0.96 & 1.79 \\
\hline \multicolumn{6}{|l|}{ Step 3} \\
\hline PHQ-9 & $-0.04(0.02)$ & 3.11 & 0.96 & 0.92 & 1.00 \\
\hline PROMIS total & $0.01(0.02)$ & 0.38 & 1.01 & 0.98 & 1.05 \\
\hline OCI-R & $-0.001(0.009)$ & 0.02 & 0.99 & 0.98 & 1.02 \\
\hline
\end{tabular}

$* p<0.001$.

CI=Confidence Interval; PHQ-15=Patient Health Questionnaire-15 Somatic Symptom Severity Scale;

SHAI=Short Health Anxiety Inventory; PHQ-9=Patient Health Questionnaire-9; PROMIS=the

PROMIS Emotional Distress - Anxiety - Short Form; OCI-R=Obsessive-Compulsive Inventory -

Revised; IUS-12=Intolerance of Uncertainty Scale - Short Form.

Gender is coded $0=$ male, $1=$ female. 